Article published in Nuclear Fusion 47 (2007) 1112

\title{
Gas Balance and Fuel Retention in Fusion Devices
}

T. Loarer 1), C. Brosset 1), J. Bucalossi 1), P Coad 2), G Esser 3), J. Hogan 4), J Likonen 5), M Mayer 6), Ph Morgan 2), V Philipps 3), V. Rohde 6), J Roth 6), M Rubel 7), E Tsitrone 1), A Widdowson2) and JET EFDA contributors*

1) Association EURATOM-CEA, CEA-Cadarache, 13108 St Paul lez Durance, France.

2) Culham Science Centre, EURATOM-UKAEA Fusion Association, OX14 3DB, UK

3) Institute of Plasma Physics, Association EURATOM-FZJ, 52425 Jülich, Germany

4) Oak Ridge National Laboratory, Fusion Energy Division, TN37831-8072, USA

5) Association EURATOM-TEKES, VTT, PO Box 1000, 02044 VTT Espoo, Finland.

6) Max-Planck-Institut für Plasmaphysik, EURATOM Association, 85748 Garching, Germany

7) Alfven Laboratory, Royal Institute of Technology, Association EURATOM-VR, Stockholm, Sweeden

*See appendix of M.L Watkins et al., Fusion Energy 2006 (Proc. 21 ${ }^{\text {th }}$ Int. Conf. Chengdu, 2006) IAEA

Email contact of the main author: thierry.loarer@cea.fr

Abstract: The evaluation of hydrogenic retention in present tokamaks is of crucial importance to estimate the expected tritium (T) vessel inventory in ITER, limited from safety considerations to $350 \mathrm{~g}$. In the framework of the European Task Force on Plasma Wall Interaction (EU TF on PWI) efforts are underway to investigate gas balance and fuel retention during discharges, and to compare the data obtained with those from post-mortem analysis of in-vessel components exposed over whole experimental campaigns. This paper summarizes the principal findings from coordinated studies on gas balance and fuel retention from a number of European tokamaks, viz. ASDEX-Upgrade (AUG), JET, TEXTOR and Tore Supra (TS). For most devices, the long-term retention fraction deduced from integrated particle balance is $\sim 10-20 \%$. This is larger than the $\sim 3-4 \%$ deduced from post mortem analysis of plasma facing components (PFCs). However, from the database available for tokamaks with their main PFCs made of carbon, the important conclusion is that the T inventory limit (set by the working guideline for operations) could be reached in ITER within fewer than 100 discharges. This, therefore, would seriously impact on operation of the device unless efficient $T$ removal processes were developed.

\section{Introduction}

Fuel retention is one of the crucial points to be investigated for next step fusion devices using Plasma facing components (PFCs) of carbon, particularly for the long discharges foreseen in ITER (400 s 7min). From the working guideline for the initial operation of ITER, the limit 
for the inventory of releasable tritium in the vacuum vessel is $350 \mathrm{~g}$. Assuming an equal mix of deuterium and tritium, a fuel injection rate of $200 \mathrm{~Pa}^{3} \mathrm{~s}^{-1}$ (or $5 \times 10^{22}$ tritium atoms per second at $293 \mathrm{~K}$ ) and a $\mathrm{T}$ retention of $5 \%$ this limit would be reached in about 70 discharges. Today, nearly all tokamaks (except a few such as Alcator-C, FTU and TRIAM-1M) are constructed with carbon used abundantly in PFCs. This enables a good data base to be assembled pertaining to fuel retention by carbon. In the framework of the EU TF on PWI, efforts are underway to investigate gas balance and fuel retention during discharges, and to compare the data obtained with those from post-mortem analysis of in-vessel components exposed over whole experimental campaigns. The aim is to assess the dominant processes of the fuel retention associated with carbon and to extrapolate the findings to ITER.

Currently, it is planned that ITER will use a carbon-fibre composite (CFC) as the material of the major PFCs at the outer and inner strike point regions in the divertor. In order to ascertain the suitability of this material, it is necessary to determine the retention associated solely with the use of carbon. This is why one of the main scientific objectives of the "ITER wall project" at JET is to "demonstrate low T retention” [1] in a fully-metallic device with a beryllium first wall and a tungsten divertor. A comparison of results with and without carbon will assist in evaluating its tritium retention characteristics. There are indications from recent analysis that the migration of carbon is very dependent on plasma conditions, and that the very high retention observed in DTE1 may in part result from the geometric differences between the MKIIA and the MKIIGB divertors [2]. From post mortem analysis, the dominant retention processes identified are direct implantation and co-deposition with carbon. The future projects with all-metal plasma facing components (AUG and JET), which replace the current carbon PFCs, should clarify the role of the latter - particularly as to the overall contribution of the carbon in the retention.

However, the net retention during plasma operations has still to be assessed as a function of the main plasma parameters $\left(\mathrm{I}_{\mathrm{p}}, \mathrm{B}_{\mathrm{T}}\right.$, plasma geometry, input power, plasma density, etc.). The gas balance method is one of the few possibilities to evaluate the fuel retention in ITER in the non-activated phase. Gas balance is regularly carried out in tokamaks [3, 8] for plasma operation of the order of 10-20 sec. Moreover, for long discharge operations carried out in machines like Tore Supra [9] and TRIAM-1M [10], this method has been demonstrated to be reliable.

Global particle balance gives insight into the exchanges of particles between the wall and the plasma, which is essential in order to understand the plasma density behaviour and control. In addition, it provides an evaluation of the wall particle inventory and its dependence 
on the plasma scenario, which is crucial for understanding the fuel retention mechanism and the extrapolation to ITER. The particle balance equation can be written as:

$$
\int_{0}^{t} Q_{\text {gas }} d t+\int_{0}^{t} Q_{N B I} d t+\int_{0}^{t} Q_{\text {pellet }} d t=N_{e}+\int_{0}^{t} \text { Vessel } d t+\int_{0}^{t} \text { Divertor } d t+N_{\text {Wall }}
$$

where $Q_{\text {gas }}, Q_{\mathrm{NBI}}$ and $Q_{\text {pellet }}$ are the particle injection rates associated respectively with gas puffing, neutral beam injection and pellet injection, $\mathrm{N}_{e}$ is the plasma fuel content, Vessel is the particle flux pumped by the pumping system of the vessel (including vessel turbo-pumps and neutral beam boxes), Divertor is the particle flux pumped by the pumps of the divertor (or limiter in the case of limiter machines) and $N_{\text {wall }}$ is the number of particles trapped or released by the wall from plasma start at $t=0$.

This balance can be evaluated at any time during a pulse, or between pulses. In principle, the only quantity not accessible to direct measurement is the relative variation of the wall inventory $N_{\text {wall }}$. The particle analysis gives the global balance over the pulse duration and thus the wall retention during transient phases of the plasma density ramp-up (dynamic retention). The method is also useful for the different plasma scenarios for ITER (eg. baseline, hybrid or advanced tokamak [11, 12]), where the plasma edge characteristics (and consequently the resulting plasma wall interaction) are significantly different and where the tokamak performance has been enhanced. The contribution of out-gassing from non-actively cooled plasma facing components can be a strong limitation in the interpretation of the gas balance and for the identification of the different retention processes. Gas balance is a reliable method for retention analysis on a short time scale, typically for discharge durations from $\sim 10 \mathrm{~s}$ to 6 minutes [9] and up to 5 h00 [10]. For longer time scales of the order of a day, this method is not yet commonly used except on devices performing long discharges (Tore Supra and TRIAM-1M). It is worth noting that on devices equipped with cryopump the method can provide a complementary measurement of the fuel retention, by comparing the integrated pumped flux evaluated by gas balance with the overall pumped flux regenerated from the cryopump at the end of the day. Indeed, the difference between the total number of particles injected during the day and the number obtained by regeneration of the cryopump after the session gives direct access to the fuel retention integrated over the day. For longer-term fuel retention analysis (weeks/months) the technique of gas balance is hampered by changing vessel conditions, particularly by events like disruptions and vessel conditioning by glow discharge cleaning. In contrast, post mortem tile and sample analysis after experimental 
campaigns is a reliable method to evaluate long-term retention, averaged over many plasma conditions and wall treatments. This method leads to lower estimates of the retention, mainly due to the impracticability of analysing more than a few percent of the PFCs of the device and also in collecting fuel-rich flakes lodged in inaccessible places. Indeed, the fuel vessel inventory deduced from post-mortem analysis is generally lower by factors of around three than the inventory derived from gas balance.

The gas balance for a number of European tokamaks, viz. AUG, JET, TEXTOR and TS, is discussed in the first part of this paper showing that the wall loading depends strongly on the operating scenario and the pulse length in steady-state conditions. An account of the global balance is reported over a full day of plasma operations. The second part of the paper reports on the analysis of post mortem samples. The third part presents the results from both methods showing that these methods are complementary since gas balance is suitable for plasma operation while the post mortem analysis integrates over all the events of a campaign (plasma, outgassing, disruptions, conditioning, etc.)

\section{Retention during the pulse}

Today, nearly all fusion devices make use of carbon in the main plasma-facing components. Consequently, several common features with respect to hydrogenic retention (also referred to as wall loading) are observed in these devices. A peak in the retention rate always occurs at the beginning of the plasma, as shown in figure 1, which displays the time evolution of the retention in long discharges in Tore Supra. Following the peak, a decreasing retention rate is observed with characteristic time ranging from 1 to $100 \mathrm{sec}$. Shorter time scales are generally observed in diverted plasmas, eg. in JET [7] and AUG [4], whilst in limiter plasmas, eg. in Tore Supra and TEXTOR, this phase is always longer. In the case of TS, this phase can extend for times up to $\sim 100$ s, as shown in figure 1 . This phase is attributed to direct implantation of ions and neutrals in a shallow surface region, with possible diffusion / migration into the bulk. The longer time to reach the second phase in limiter devices is attributed to both a lower particle fluence at the plasma edge than in the divertor region and a higher edge electron temperature in the limiter scrape-off layer (70-100eV), leading to deeper implantation [13] compared with the $10-30 \mathrm{eV}$ in the divertor. However, the major portion of these particles is retained temporarily (“dynamic retention”) during the plasma pulse and released after the shot. This retention tends to saturate with time and implantation fluence, even if recent results suggest a longer time constant for saturation. The number of particles loaded into the wall during this transient phase is material dependent, as observed in JET 
when using limiters of beryllium then carbon. It was found that the gas injection required to reach a given plasma density was significantly higher ( 3-4) with beryllium than with carbon PFCs [14]. However, at the end of the pulse the particles released were also enhanced by the same factor. This dynamic retention is not influenced by the discharge history of the retention and is reproducible when the same pulse is repeated. Special wall treatments such as glow discharge cleaning (GDC) can change this reservoir, but only in a transient way that is not reproducible. This retention is thus independent of the plasma conditions such as the plasma current, the total input power, the heating scenario, the fuelling method and also the flat top duration ( $>6 \mathrm{~min}$ in Tore Supra). However, the associated inventory is recovered at the end of the pulse, as discussed in section 3.

In contrast, the retention observed during the second phase depends both on the plasma conditions and pulse duration. Indeed, in Tore Supra, for long ( $>1 \mathrm{~min}$ ) and steady-state discharges a constant retention rate is observed. The fraction of the injected gas retained can be as high as $50-60 \%$, the total retention being proportional to the length of the plasma pulse. For low absolute fuelling, however, the retention is found to be close to zero. This is also illustrated for AUG in figure 2, where the total number of particles pumped out during the pulse, and up to $12 \mathrm{sec}$ after its end, is displayed as a function of the total number of particles injected during the pulse. It is worth noting that the total D pumped out also includes the major part of the out-gassed flux released at the end of the pulse and which corresponds typically to the dynamic retention. However, as discussed later in the paper, the number of particles recovered at the end of the pulse is more or less constant within a factor of two. In any case, this corresponds to a very minor part (maximum of $25-30 \%$ for the lowest gas injection cases and down to $10-15 \%$ for medium particle injection) in the overall balance. In figure 2, a significant retention is observed unless low fuelling rates are used. Indeed, for low absolute fuelling, where the plasma density is close to the natural density (plasma density built up by the recycling flux only, in the absence of any external fuelling), the retention is shown to be close to zero. For injection lower than $\sim 3 \times 10^{22} \mathrm{D}$, the gas recovered after the pulse is at least equivalent to the input. This is independent of the dominant heating (NBI or ICRH) with no significant influence by the tungsten coverage increasing between 2003 and 2005 (from 45 to 70\% W coverage, respectively). This retention is attributed to co-deposition of hydrogen with eroded wall material forming hydrogen rich co-deposits in locations not "accessible” by plasma operation and / or the usual conditioning / de-conditioning mechanisms (GDC, disruptions, etc.). This is considered as the most dominant "long term" fuel retention mechanism since the growth is linear with the discharge duration. 
In AUG, typically $75 \%$ of the injected gas is exhausted during and between shots. Another $10 \%$ is pumped during long periods (nights / weekends), leading to an estimated retention deduced from gas balance of 10 to $20 \%$. The wall acts as an important dynamic particle sink / source; in high density discharges $\mathrm{D}$ is stored in the vessel and for low density discharges the net vessel inventory can be only weakly decreased as discussed later. The density range of the plasma discharges in AUG requires a gas injection of between $5 \times 10^{20}$ and $5 \times 10^{21} \mathrm{Ds}^{-1}$, which leads to a net retention of 0.5 to $1 \times 10^{21} \mathrm{Ds}^{-1}$ for the strongest retention. Recent results from JT-60U [15] for type III ELMy H-modes also show that with very low gas injection $\left(1.0 \times 10^{21} \mathrm{Ds}^{-1}\right)$, for a plasma density of 0.64 the Greenwald density $\left(\mathrm{n}_{\mathrm{GW}}\right)$, the global particle balance reached equilibrium during the pulse and the retention was close to zero. The rate of gas exhaust by the divertor cryopump closely matches the injection rate for a constant plasma density, thereby confirming a retention close to zero. However, for higher density up to $0.82 \mathrm{n}_{\mathrm{GW}}$, a stronger fuelling rate (up to $10^{22} \mathrm{Ds}^{-1}$ ) is applied and, as observed on AUG and JET, a steady state retention of $\sim 2 \times 10^{21} \mathrm{Ds}^{-1}(\sim 20 \%$ of the total injection: NBI + Gas) is observed during the pulse. When the gas recovered between pulses is taken into account in the overall balance for a full day of experiments, this value is reduced but the outgasing does not compensate the overall retention observed during the pulse. The contribution of the recovered gas in the overall balance is discussed later.

Previous experiments in Tore Supra and in DIII-D [16, 17] have been reported, showing that the wall loading could be controlled by active pumping. However, it is worth noting that these results are comparable to the reported effects of low fuelling as shown above for AUG, JET and JT-60U and that the number of particles recovered during these discharges is of order the dynamic retention observed during the first part of the pulse. Indeed, in all these experiments the retention is shown to be negligible and even negative when the gas injection is low and the density close to the natural density. The surfaces depleted during these experiments were the main area of interaction with the plasma (limiters for Tore Supra and target plates of the divertor for DIII-D) whilst the retention process, such as co-deposition, is not modified by the absence of fuelling and / or pumping.

The active pumping has no effect on the retention as demonstrated by the comparison of two similar long discharges (1min) carried out in Topre Supra with and without active pumping [18]. The retention rate during the pulse and the overall balance at the end of the two discharges was the same within the error bars. The feedback control on the gas injection to obtain the target density was higher with active pumping by the amount exhausted by the pumps, showing that the effect of the active pumping on the wall loading is totally negligible 
[18]. Also, different fuelling methods by gas injection, supersonic jet [18] and pellet [19] injection have been investigated in Tore Supra and, up to now, no difference has been noticed in terms of retention.

It is also worth noting that no influence of ELMs has been observed up to now on the global particle balance. However, the carbon transport at the edge is known to strongly depend on the location of the power deposition particularly during transient events like ELMs. Indeed, analysis of quartz microbalance data (QMB) mounted in the inner divertor louvre region in JET confirmed that the carbon deposition is significantly higher for H-modes than L-modes [20]. Also, plasma configuration is the most important factor determining the local material deposition at the QMB. The difference in carbon deposition between $\mathrm{H}$ and $\mathrm{L}$ mode is less pronounced when the strike-points are located on the vertical tiles. The largest deposition (up to $\sim 5 \cdot 10^{16} \mathrm{C}$ atoms $/ \mathrm{cm}^{2} \mathrm{~s}$ ) occurs in ELMy $\mathrm{H}$ mode shots on the inner divertor $\mathrm{QMB}$, if the strike-point is located on the inner base tile with line of sight to the QMB [20, 21].

For short plasma discharges, the particle balance is dominated by the dynamic retention and the main processes that contribute to particle retention (shallow and deep implantation, co-deposition and migration/diffusion) are difficult to separate under those conditions [5]. Long discharges (>1 min) in TS, indicate that the retention is proportional to the fluence. This effect was already observed in the first long discharges performed in Tore Supra $\left(\mathrm{I}_{\mathrm{p}}=1.0 \mathrm{MA}, \mathrm{B}_{\mathrm{T}}=3.9 \mathrm{~T}\right)$ with the plasma leaning on the graphite inner wall [22] before the upgrade to the CIEL project (Toroidal Pump Limiter). For these experiments, the retention rate for three consecutive discharges (without any conditioning) was $\sim 3.0 \times 10^{20} \mathrm{Ds}^{-1}$, and equal to the gas rate (no active pumping) during the steady state phase of the discharge ( $\sim 0 \mathrm{sec}$ ). However, the mechanism is not only determined by co-deposition but also possibly by diffusion / migration of deuterium in carbon porosities and cracks. This is supported by analyses of carbon deposits originating from different locations inside the vessel. These reveal relatively low deuterium content and are unable to account for the large deuterium invessel retention that was derived from particle balance analysis [23]. Recent laboratory [24] data on deep penetration of hydrogen in CFC support this but more work is needed for a definitive conclusion. In Tore Supra with the new toroidal CFC pump limiter, analysis of the retention during long discharges heated with Lower Hybrid Current Drive (LHCD) still indicate a strong dependence of the retention rate on the LHCD power, as shown in figure 3 [25]. This effect is not observed for Ion Cyclotron Resonance Heating (ICRH), independently of the ICRH frequency and phase conditions. Up to now in Tore Supra, this is the only 
significant dependence observed for retention. At medium density, no dependence on the edge plasma characteristics was found (Te at the LCFS is in the range $30-100 \mathrm{eV}$ ), irrespective of the fuelling method (pellets, gas injection) or the radiated fraction. A possible explanation could be the enhancement of the retention rate by a modification of the SOL, induced by the supra-thermal electrons generated in front of the LH grill [25]. So far, no dependence of the retention on the LH input power has been observed on other machines using LHCD as heating systems, such as JET and JT-60U.

In the second phase of pulses in non-actively cooled devices, a strong plasma density increase is reported when the increase in PFC temperature during the shot turns the wall from a net sink to a net source. This behaviour is called wall saturation by some authors. This is the case in JT-60U, where the effect does not occur in short pulses $(<15$ s), but appears progressively in repetitive long discharges [8]. The behaviour was also observed for repetitive discharges in TS before the CIEL upgrade, as shown in figure 4 [26] for a series of long discharges with LHCD heating only, and when "only" 80\% of the PFCs were actively cooled. In the absence of an gas injection, the slow increase of the plasma density is attributed to a moderate temperature increase of $\sim 20^{\circ} \mathrm{C}$ on the non-actively cooled area. This shows that with non-actively cooled PFCs, the thermal out-gassing flux is not constant and it becomes non negligible for long discharges and / or high energy experiments. When the out-gassed flux exceeds the total exhaust capability, the density control is also lost in JT-60U in spite of active pumping by the divertor cryopump. In any case, this strong out-gassing does not prevent retention from occurring in areas not directly heated / viewed by the plasma outflux (eg first wall, gaps between tiles, areas below the divertor, etc.). As a consequence, the socalled wall saturation is a paradox in terms of global particle balance since the strong heating of the target plates results in them becoming "non-saturated". It is worth noting that in "fully" actively cooled devices (in Tore Supra 98\% of the PFCs of the CIEL project are actively cooled) as shown in figure 1, the out-gassing source is controlled / constant, the retention rate is constant, and no "history effect" is observed for the same plasma parameters. The total increase of the vessel inventory after discharge \#32299 was $7.5 \times 10^{22} \mathrm{D}$ (outgassing at the end of the discharge taken into account) and was much larger than what could be expected [13] from saturation of the carbon PFCs $\left(\sim 5 \times 10^{21} \mathrm{D}\right)$. Finally, after a series of three repetitive long discharges, similar to the one displayed in figure 1, and accumulating 15 min of plasma operation, the retention rate has been found to be constant and reproducible (\#32300 fig.1) from shot to shot without any sign of wall saturation. 


\section{3. - Recovery between pulses}

The out-gassed flux during, and particularly after, the pulse is a significant contribution to the overall gas balance associated with a short pulse. Figure 5 shows the total recovery of D integrated over $600 \mathrm{~s}$ from the end of a shot as a function of the total D injected during the pulse, for a series of non-disrupting JET pulses with the MKII_SRP and MKII_HD divertors. The duration of $600 \mathrm{~s}$ ensures that the out-gassing flux is not underestimated. The long term recovery (nights / weekend) in the absence of any conditioning is very weak (a few \% of the total recovered during the experimental day between pulse [6]) compared to the amount recovered $\sim 10$ min after the pulse. In any case, this amount does not vary significantly with the divertor type and is always larger than the plasma content $\left(2-6 \times 10^{21} \mathrm{D}\right)$, showing that the wall does trap and release particles transiently (dynamic retention). In figure 5, the solid line represents a full recovery of the particles at the end of the pulse when the balance during the pulse is taken into account. It can be seen that for low fuelling equilibrium can be reached, but as the plasma density is increased by gas injection the retention also increases whilst the recovery does not increase by more than a factor of two. The number of particles recovered after the end of the pulse does not depend strongly on the discharge characteristics, such as the fuelling rate, fuelling method, plasma current, flat top duration, power input, heating scenario or plasma configuration: the quantity of $\mathrm{D}$ released after a non-disruptive discharge is largely independent of the discharge history (see figure 5). The same behaviour was previously observed when JET was operating with limiters [14] and similar results are found in AUG (45\% W coverage) based on the D recovery by He GDC after the discharge [4] and in Tore Supra [5, 18, 27] with actively cooled PFCs. It is worth noting that since the retention increases with the total number of particle injected (see figure 2) the recovery of the particles trapped during the pulse cannot be complete since the out-gassing corresponds to the dynamic retention observed at the beginning of the discharge (phase 1).

The overall integrated balance extended after the end of the pulse to include the outgassed flux shows that the retention fraction is low for short pulses, while it is equivalent to the retained fraction during the pulse for long discharges, where the recovery after the shot becomes negligible compared to the wall inventory accumulated during the shot. This is clearly seen when performing particle balance integrated over longer periods ( $\sim 10$ hours) as shown in figure 6 which compares particle balance performed for a series of short pulses ( $550 \mathrm{~s}$ of accumulated plasma time in 24 discharges) and a series of long pulses ( $1330 \mathrm{~s}$ of accumulated plasma time in "only" 7 discharges) on Tore Supra. For short pulses, the inventory accumulated over the day can be recovered by a night of He glow discharge 
cleaning ( $5 \times 10^{22} \mathrm{D}$ ), yielding an overall balance close to equilibrium for short pulses as shown by the "blue" area at the bottom of figure 6 . In contrast, long discharges lead to a significant inventory build up which is proportional to the plasma duration and which becomes much larger than the possibility of overnight He GDC recovery. Finally, for both series the contribution of the recovery to the overall balance is nearly the same (contributions of out-gassing for the series of long and short discharges cannot be easily distinguished in figure 6) and becomes negligible in the overall balance. However, it is worth noting that the evaluation of the retention by gas balance analysis cannot separate the trapping process for the particles: shallow and deep implantation, retention in gaps, bulk migration or co-deposition. On the other hand, the He GDC can only remove particles from areas which it can "view" and where the implantation is shallow. In cases of deep implantation, migration into the bulk or co-deposition particles cannot be removed by classical cleaning discharges. Indeed, He or $\mathrm{H}$ plasmas and He GDC have been demonstrated to have a very low efficiency in removing the T retained in JET from the DTE experiments $[6,28]$. Therefore, the comparison of the gas balance for a series of short and long discharges suggests that the main processes for long term retention are dominated by deep implantation (including D migration into the bulk) and co-deposition.

Accuracy in gas balance studies is, however, limited by the requirement to obtain the differences between large numbers, particularly when extended over periods as long as days or even weeks of operation (pulse $10-20$ sec compared to a day $10^{5} \mathrm{sec}$ ). The gas balance accuracy depends strongly, therefore, on the duration over which the neutral pressure is integrated. Moreover, the difficulty in assessing the overall contribution of hydrocarbons during plasma discharges, between discharges and also after a disruption adds to the uncertainty. Therefore, the gas balance probably represents the upper limit for the evaluation of the retention.

\section{Sample analysis in AUG, JET and Tore Supra}

For long term retention integrated over an experimental campaign, post-mortem analysis is a proven method to assess the retention. However the results are integrated over a full experimental campaign which includes various events: different plasma scenarios, conditioning procedures and disruptions. The method is commonly used in almost all the machines in which long term retention is studied. In JET, divertor tile analysis after the MkIIGB divertor campaign (total duration in divertor configuration of $57500 \mathrm{sec}$ ( 16h00 of plasma) [29] found a C deposition of about $400 \mathrm{~g}$, mainly in the inner divertor. This leads to a 
retention rate of $3.4 \times 10^{20} \mathrm{Cs}^{-1}$. During these plasmas a total amount of $766 \mathrm{~g}$ of $\mathrm{D}$ has been injected corresponding to an integrated ion flux on the inner target of $1.3 \times 10^{27}\left(\sim 6-7 \mathrm{~m}^{2}\right)$. In the deposits on the inner divertor the $\mathrm{D} / \mathrm{C}$ ratio was as high as 0.2 and the overall $\mathrm{D}$ retention estimated from these data is about 4\% (34g) of the total amount injected. However, only samples from the divertor have been included in this analysis. Neither tile gaps, nor inner wall guard limiters have been taken into account. Thus, the retention fraction of $4 \%$ represents a lower limit to the overall retention and corresponds to an averaged retention of $2.6 \times 10^{20} \mathrm{Ds}^{-1}$.

Recent analysis of tiles from the MKII-SRP divertor in JET shows that the total integrated fuel (deuterium retention) in the divertor, in co-deposited layers, is about $42 \mathrm{~g} \mathrm{D}$ compared with an integrated fuel injection of $1800 \mathrm{~g}$ D. This yields a retention fraction of $2.7 \%$ in the divertor, similar to that observed in previous campaigns. The number represents a lower limit since it does not include retention in the main chamber, the private flux region tile (still to be analysed), tile gaps and so on. However, based on previous data, the additional retention expected from those areas is not expected to increase the present value by more than $50 \%$, yielding an overall long-term D retention of about 4\% in the 2003 - 2004 JET experimental campaign.

Careful sample analysis has also been carried out in AUG to study the deposition of D, B and $\mathrm{C}$ in order to assess the effect of increasing the area covered with $\mathrm{W}$ [30]. Coated divertor tiles (150 nm of Re and $3 \mu \mathrm{m}$ of $\mathrm{C}$ ) have been analyzed before and after plasma exposure using Rutherford back scattering (RBS) to measure metallic concentrations and nuclear reaction analysis (NRA) to evaluate the D concentrations. A total of $2.4 \mathrm{~g}$ of $\mathrm{D}$ has been collected from the inner and outer divertor but also from below the divertor and behind the wall. Figure 7 shows a cross section of the inner divertor showing the $\mathrm{D}$ deposition as a function of the location (tiles). When integrated over the toroidal direction, and assuming a uniform deposition, this corresponds to $3.1 \%$ of the $\mathrm{D}$ input. This amount can be increased to $3.1 \mathrm{~g}(4.1 \%)$ when assuming the same $\mathrm{D} /(\mathrm{B}+\mathrm{C})$ ratio of 0.4 on the two vertical targets of the inner divertor. Optical inspection indicates that retention in tile gaps is probably small. For the 2002/2003 campaign, AUG was mainly a carbon machine with a retention governed by trapping on the inner divertor tiles, where $70 \%$ of the trapped inventory was found. A further $20 \%$ was found in remote areas (eg. below roof, on baffles, etc.). An overall retention of $~ 4 \%$ of the total input results, compared to 10 to $20 \%$ from the gas balance analysis.

The 2004 / 2005 campaign, during the transition period to a full W machine (divertor and some limiters were still carbon) did not exhibit a significant difference in the retention 
compared to 2002 / 2003. During the 1995 / 1996 campaign of AUG, with carbon in the main chamber and $\mathrm{W}$ in the divertor, a retention of $2 \%$ of input has been estimated from sample analysis [31], similar to recent values.

In Tore Supra, gas balance integrated over a full campaign shows that $\sim 25 \%$ of the injected gas is retained corresponding to an averaged retention of $10^{20} \mathrm{Ds}^{-1}$, while only $3 \%$ was found in the deposited layers analysed so far [23] and would correspond to about $10^{19} \mathrm{Ds}^{-}$

${ }^{1}$. This analysis has been carried out on deposited carbon layers removed from the leading edges of the neutralizer, the underside of the toroidal limiter, the lateral faces of the antenna protection and from the outboard limiter. The D / C ratio observed in these layers is typically lower then $10 \%$ and the integrated retention in these layers counts for only $10 \%$ of the D retained in Tore Supra. A possible candidate for the retention is carbon deposited in the gaps between the fingers of the limiter and possible flakes below the limiter. Finally, as suggested by the constant retention rate during the long pulse operations, and the dependence on LHCD, bulk migration and/or diffusion into porous CFC could also play a role. Indeed, laboratory experiments have shown a deep penetration of D into CFC, far beyond the ion implantation range. The fraction retained does not saturate, as is the case with graphite, but increases with the square root of the fluence [24]. However, up to the present, despite the efforts expended at TS consistency has not been achieved in the evaluated particle balance.

\section{Discussion}

For various devices, the long term retention fraction evaluated from integrated gas balance ( 10-20\%) is larger than the retention deduced from post mortem analysis of PFCs which is $\sim 3-4 \%$. However both these methods are subject to large error bars. It is difficult to determine the contributions over a full experimental campaign, from D recovery from wall conditioning, disruptions or out-gassing over long periods (compared to plasma operation). For gas balance performed typically for an experimental day or week, the accuracy depends on the integrated difference between the injection and the exhaust. The contribution of non-hydrogenic species (such as hydrocarbons) is another difficulty. In contrast, post-mortem analysis tends to underestimate the long-term retention, as it can only be performed on a restricted set of samples representing only a few percent of the overall surface exposed to the plasma. The results are then extrapolated to the whole device, assuming toroidal symmetry, and have difficulties in accounting for retention in areas such as limiters, other large areas in the main chamber, gaps between tiles, etc. In AUG, the long-term retention deduced from gas balance is $10-20 \%$ of the injected particles, while it is estimated to be only $3-4 \%$ from post mortem 
analysis. In JET, post-mortem analysis also yields about a 4\% retention rate, but gas balance integrated over experimental campaigns indicates retention of $6 \%$, with no noticeable influence of the wall temperature $\left(320\right.$ or $200^{\circ} \mathrm{C}$ ) [32]. During the DT experiments in TFTR [33] and in JET (1997-1998) [34], the immediate T retention has been shown to be about 40\% during the campaign, while 17\% (6g T) remained after intensive cleaning procedures executed after the D-T experiments in JET. Only 3g were recovered in the sample analysis, suggesting that the missing $3 \mathrm{~g}$ would be found in flakes located in the sub-divertor volume, out of an estimated total amount of $1 \mathrm{~kg}$ of flakes [35]. The retention in the tiles of the divertor was evaluated to be about $2 \%$, showing that values deduced from post-mortem analysis tend to underestimate the retention mainly due to the impracticability of analysing the complete surface of the device and also of finding all the flakes.

The fuel retention in carbon devices can be attributed to four mains processes: adsorption, implantation, bulk migration (including diffusion) and co-deposition. The first two processes saturate with plasma pulse length and the retention rate observed in phase 1 can be explained by adsorption. Indeed, it has been shown on JET and Tore Supra that the recovery after the discharge is well correlated with the inventory accumulated during phase 1 . This suggests that this retention mechanism saturates during the plasma pulse and that this process is transient since these particles are recovered at the end of the pulse. As no wall saturation is observed for long discharges in steady state and in actively cooled machines, the main long term retention processes are not expected to be related to adsorption or implantation. Indeed, implantation is a permanent mechanism with a strong chemical bond between $\mathrm{C}$ and $\mathrm{D}$ which saturates when a maximum concentration is reached [13]. The fuel retained by implantation cannot be released under steady state operation and special techniques such as local heating and / or conditioning are required.

Bulk migration (including diffusion) and co-deposition are identified as the dominant mechanisms for fuel retention, since no saturation processes limit the potential number of particles to be retained in the vessel. Co-deposition is linked to the carbon source and is a permanent mechanism (chemical bonds). It is thought to be the main mechanism for the retention observed in JET for the D-T experiments in 1997-1998; $3.7 \mathrm{~g}$ of T were still left in the vessel after an intense cleaning campaign. "Only" $0.2 \mathrm{~g}$ was found when analysing a part of the divertor and main chamber tiles, confirming that implantation in areas exposed to plasma interaction was not the dominant mechanism for retention. However, it is worth noting that recent results for CFC materials exhibiting a retention in the bulk by migration, increasing as the square root of the fluence, could enhance the long term retention process 
[24]. For these D-T experiments in JET, flakes ( 3g in sub-divertor flakes [35]) found in remote shadowed areas suggest that co-deposition was the dominant process of retention.

There are large uncertainties in the predicted retention in ITER, based on an extrapolation from current devices with carbon PFCs [5]. However, the adsorption processes should involve around $0.5 \mathrm{~g}$ of $\mathrm{T}$, which will be released after the discharge. Also, the implantation should involve a limited amount of $\mathrm{T}$, in the range of 5 to $40 \mathrm{~g}$, before reaching saturation. Migration in the bulk and co-deposition are at the moment the major concerns as, in carbon dominated devices, the retention linked to these process increases with the particle fluence.

Assuming similar retention fractions in ITER as in present carbon-dominated devices, an averaged retention of $10 \%$ and assuming a gas puff rate of $200 \mathrm{Pam}^{3} \mathrm{~s}^{-1}$ for 50-50\% of D-T, $\left(5 \times 10^{22} \mathrm{Ts}^{-1}\right)$ yields an equivalent long-term retention of $10 \mathrm{~g}$ of $\mathrm{T}$ per $400 \mathrm{~s}$ discharge. This limits the number of discharges to 35 before reaching the working guideline limit of $350 \mathrm{~g}$, while a retention of $3 \%$ would increase this number to 115 (1\% retention of the T injected in a 400 s ITER discharge would lead to a retention of $1 \mathrm{~g}$ per discharge independently of the material). The current selection of ITER materials (C, W and Be) should lead to a somewhat lower inventory compared to a full $\mathrm{C}$ machine, as most of the retention processes identified so far are linked to carbon (implantation of D in C, deep diffusion / migration and trapping of D in $\mathrm{C}$, co-deposition of $\mathrm{D}$ with eroded C). However, there is presently no experimental data from tokamaks with the material mix contemplated for ITER to assess this effect, and modelling is still at the early stage: large uncertainties remain in the predicted $\mathrm{T}$ retention rates.

\section{Conclusion}

Gas balance and fuel retention analysis carried out in AUG, JET, TEXTOR and Tore Supra, show that the vessel retention depends strongly on the scenario and the pulse length in steady state operations. For longer-term fuel retention studies (weeks / months) analysis by gas balance is hampered by changing vessel conditions, in particular events such as disruptions and vessel conditioning by glow discharge cleaning. However, gas balance is one of the few techniques to evaluate the fuel retention in ITER in its non-activated phase. Post mortem tile and sample analysis after experimental campaigns is a complementary method to evaluate long term retention, averaged over many conditions and wall treatments but performed for a restricted area. The resulting long-term retention fraction from integrated particle balance is $\sim 10-20 \%$ and is larger than the values of 3-4\% deduced from post mortem analysis of PFCs. Also, up to now, no significant influence on the global balance has 
been observed with increasing W coverage (from 45 to $70 \%$ of W in AUG), nor is the balance influenced by ELMs. Finally, from the database available from tokamaks with carbon as the main PFCs, the important conclusion is that, assuming a similar retention to that in carbondominated devices, the T inventory limit in ITER could be reached within $~ 100$ discharges, unless efficient $\mathrm{T}$ removal processes are developed.

\section{Acknowledgements}

The authors would like to thank the referees for valuable remarks and suggestion for improving the quality of the paper. 


\section{References}

[1] A LIOURE et al., "JET Enhancement Programmes: More heating power and diagnostic capabilities in preparation for ITER”. Fusion Eng. Design 74, Issues 1-4, November 2005, Page 141-146.

[2] J.P COAD et al., "Overview of material re-deposition and fuel retention studies at JET with the Gas Box divertor”, Nucl. Fusion, 46 (2006) 350-366.

[3] M MAYER et al., “Hydrogen inventory in Fusion devices”, J of Nuclear Materials, 290293 (2001) 381-388.

[4] V MERTENS et al. "Hydrogen gas balance in ASDEX Upgrade with DIV IIB", $30^{\text {th }}$ EPS Conference on Controlled Fusion and Plasma Physics. St Petersburg, 7-11 July 2003, ECA, Vol.27A, P-1.128

[5] E TSITRONE, “Key plasma wall interaction issues towards steady state operation”, J of Nuclear Materials, 363-365, (2007) 12-23.

[6] T LOARER et al., “Tritium Particle Balance and retention during DT discharges in JET”, J of Nuclear Materials, 337-339 (2005) 624-628.

[7] T. LOARER et al., "Fuel retention over a full day of experiments in JET” Fusion Science and Technology, Volume 48, Number 1, July/August 2005, Page 306-309.

[8] N ASAKURA et al., "Wall pumping and saturation in divertor tokamaks", Plasma Phys. Control. Fusion 46 (2004) B335-B347.

[9] D VAN HOUTTE et al., "Recent fully non-inductive operation results in Tore Supra with 6 min, 1 GJ plasma discharges ”, Nucl. Fusion 44, (May 2004) L11-L15.

[10] M SAKAMOTO et al., "Multiscale Phenomena of Plasma-Wall Interaction in Long Duration Discharges on TRIAM-1M”, Fusion Energy 2006 (Proc. $21^{\text {st }}$ Int. Conf. Chengdu, 2006) (Vienna: IAEA) CD-ROM file [EX/P4-25] and http://www-naweb.iaea.org/napc/physics/FEC/FEC2006/html/index.htm [11] ITER Physics Basis 1999, Nucl. Fusion 39, 2137.

[12] A SIPS et al., “Advanced scenarios for ITER Operations”, Plasma Phys. Control. Fusion 47 (59) (May 2005), pp A19-A40.

[13] K WILSON et al., Nucl. Fusion, Vol.1, “Atomic and Plasma material interaction data for fusion”, Volume 1, p. 31, 1991.

[14] R SARTORI et al., "Deuterium release measurements in the Be phase of JET and determination of tritium content in JET”, J of Nuclear Materials, 176-177 (1990) 624-629. 
[15] H KUBO et al., "Particle Control under Wall Saturation in Long-pulse High-density Hmode Plasmas of JT-60U”, Fusion Energy 2006 (Proc. 21 ${ }^{\text {st }}$ Int. Conf. Chengdu, 2006)

(Vienna: IAEA) CD-ROM file [EX/P4-11] and http://www-naweb.iaea.org/napc/physics/FEC/FEC2006/html/index.htm

[16] T LOARER et al., "Particle exhaust experiments with pump limiter, ergodic divertor and vented limiter in Tore Supra”. Plasma Phys. Control. Fusion 37 (1995), pp A203-A214.

[17] R MAINGI et al., "Control of wall particle inventory with divertor pumping on DIII-D“, Nucl. Fusion, 36, N² (February 1996) pp 245-253.

[18] B PEGOURIE et al., "Long discharge particle balance and fuel retention in Tore Supra”, Physica scripta, T 111 (2004) p 23.

[19] A GERAUD et al., "Pellet fuelling in Tore Supra long discharges", J of Nuclear Materials, 337-339 (2005) 485-489.

[20] G ESSER et al., "Effect of plasma configuration on carbon migration measured in the inner divertor of JET using quartz microbalance”, J of Nuclear Materials, 337-339 (2005) 8488.

[21] A KIRSCHNER et al., "Material erosion and redeposition during the JET MkIIGB-SRP divertor campaign”, Fusion Energy 2006 (Proc. 21 ${ }^{\text {st }}$ Int. Conf. Chengdu, 2006) (Vienna: IAEA) CD-ROM file [EX/3-5] and http://www-naweb.iaea.org/napc/physics/FEC/FEC2006/html/index.htm .

[22] D VAN HOUTTE et al., “One minute pulse operation in the Tore Supra tokamak”, Nucl. Fusion 33, 137 (1993).

[23] C BROSSET et al. "Deuterium concentration is deposited carbon layers in Tore Supra". J of Nuclear Materials, 337-339 (2005) 664-668.

[24] J ROTH et al., $17^{\text {th }}$ Conference on Plasma Surface Interaction, "Deuterium retention in carbon fibre composites NB31 and N11 irradiated with low-energy D ions”, J of Nuclear Materials 363-365 (2007) 822-826.

[25] J BUCALOSSI et al. "Particle control in high power, high density long pulses on Tore Supra”, $32^{\text {nd }}$ EPS Conference on Plasma Phys. Tarragona, 27 June -1 July 2005, ECA, Vol.29C, O-4.005 (2005).

[26] C GRISOLIA et al., "Plasma wall interaction during long pulse operation in Tore Supra”, J of Nuclear Materials, 266-69 (1999) 146-152.

[27] J BUCALOSSI et al., "Deuterium in-vessel characterisation through the use of particle balance on Tore Supra”, J of Nuclear Materials, 363-365, (2007) 759-763 
[28] J.P COAD et al., “Carbon Deposition and Hydrogen Isotope Retention in JET”, Physica Scripta. Vol. T81, 7-12, 1999.

[29] J LIKONEN et al., "Berylium accumulation at the inner divertor of JET”, J of Nuclear Materials, 337-339 (2005) 60-64.

[30] M MAYER et al., "Carbon Erosion and Deposition on the ASDEX Upgrade Divertor Tiles”, J. Nuclear Materials 337 - 339 (2005) 119-123.

[31] D SCHLEUSSNER et al., "Hydrogen isotope inventories in the ASDEX-Upgrade tungsten coated divertor tiles”, J of Nuclear Materials, 266-269 (1999) 1296-1302.

[32] J BUCALOSSI et al., "Particle balance studies in JET”. $28^{\text {th }}$ European Conference on Controlled Fusion and Plasma Physics, Funchal, 18-22 June 2001, ECA, Vol.25A (2001) 1629-1632.

[33] C SKINNER et al. "Plasma wall interaction and tritium retention in TFTR”, J of Nuclear Materials, 241-243 (1997) 214-226.

[34] P ANDREW et al., “Tritium recycling and retention in JET”. J of Nuclear Materials, 266-269 (1999) 153-159.

[35] N. BEKRIS et al., “Characterisation of flakes generated in JET after DD and DT plasma operations” J of Nuclear Materials, 337-339 (2005) 659-663. 


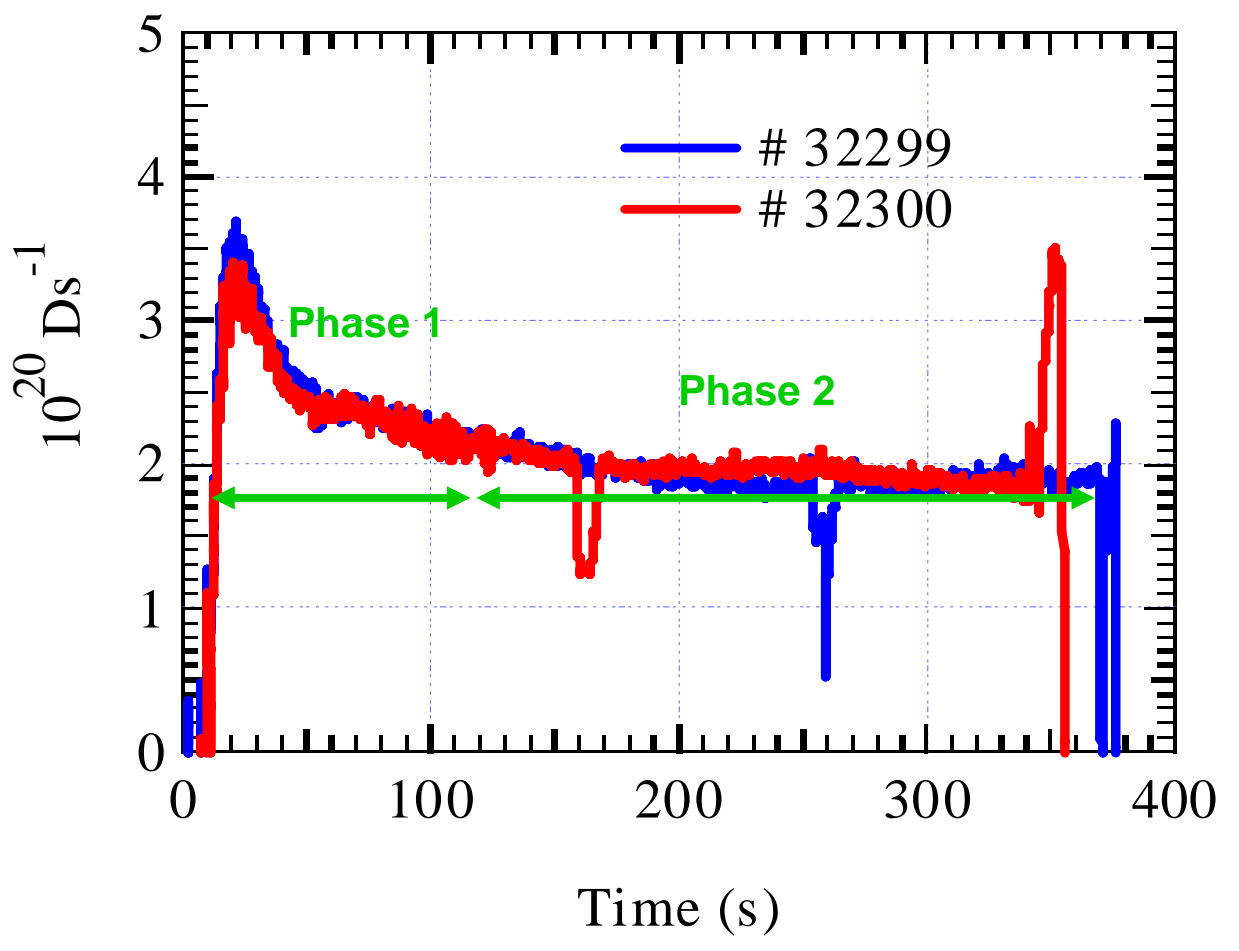

Figure 1: Retention as a function of time for two consecutive long discharges in Tore Supra. 


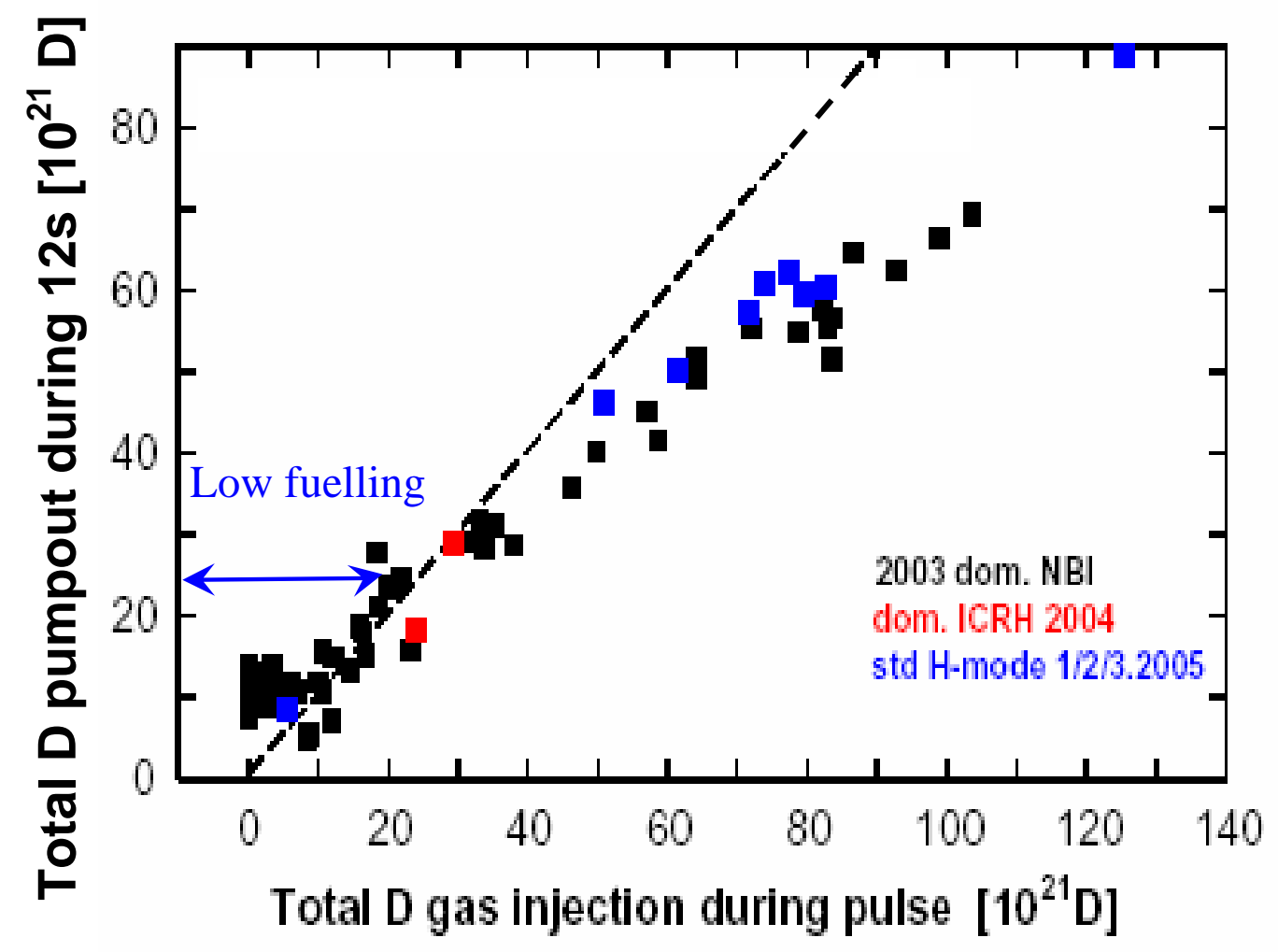

Figure 2: Particle recovery as function of total D injected in AUG. Different plasma scenarios and different $\mathrm{W}$ coverage are described in the text. 


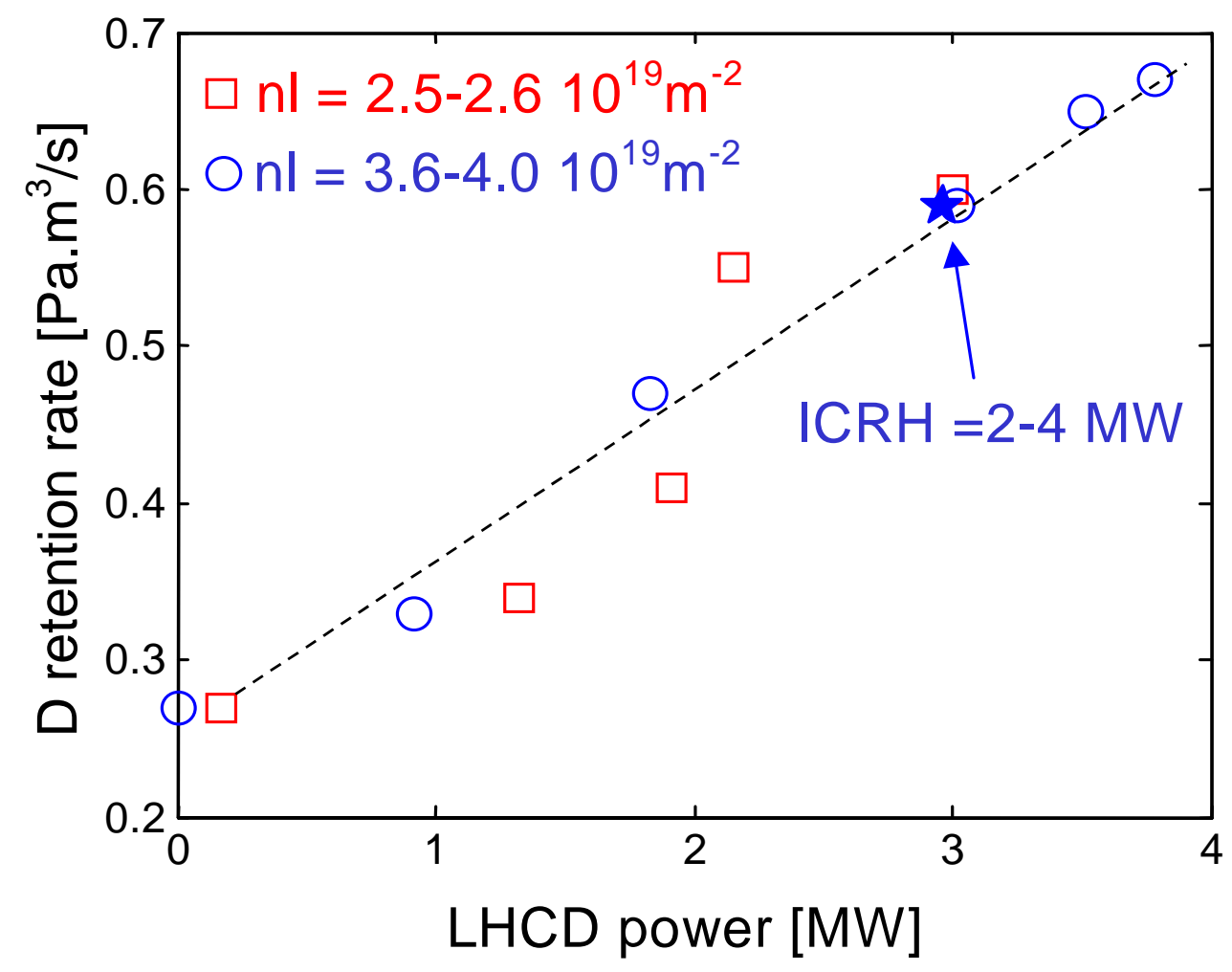

Figure 3: D retention rate as a function of LHCD power, for different plasma densities and ICRH power in Tore Supra [25]. 


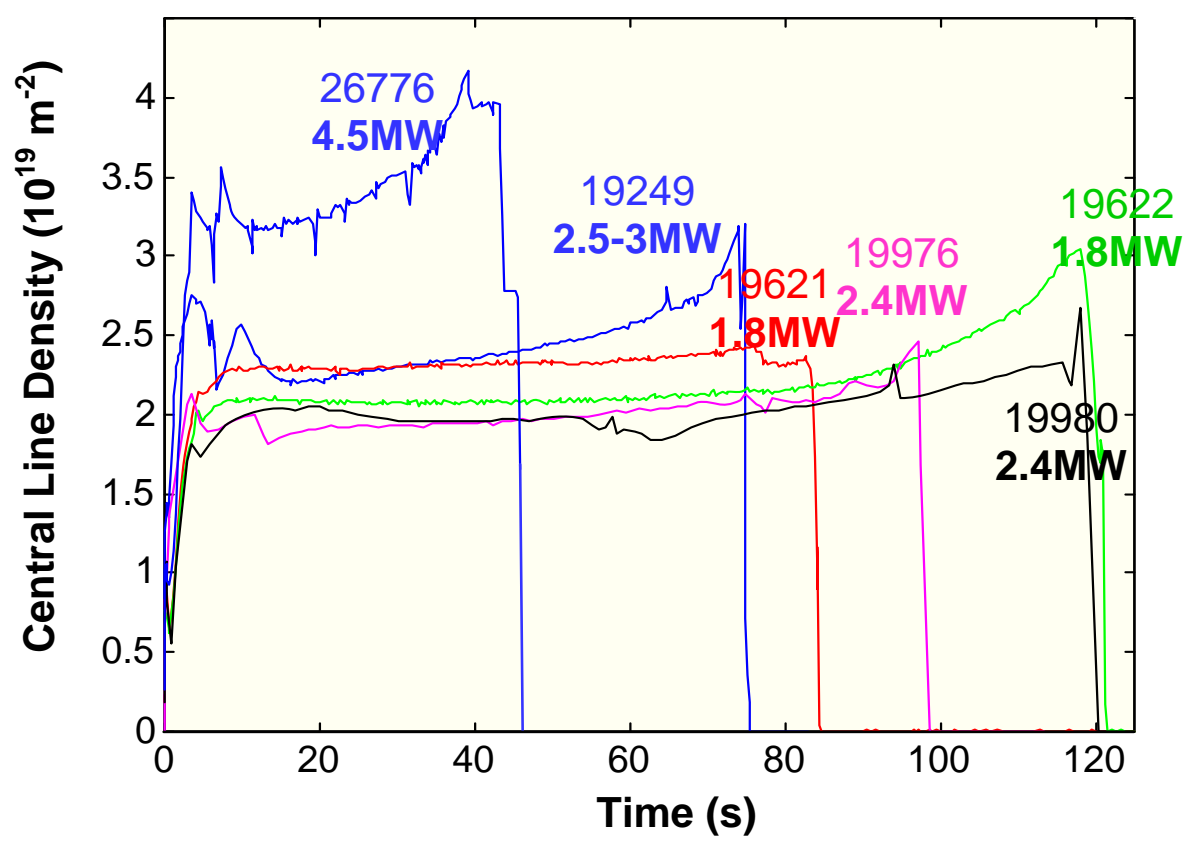

Figure 4: Time evolution of long discharges in Tore Supra, with $80 \%$ of plasma facing components actively cooled. [26]. 


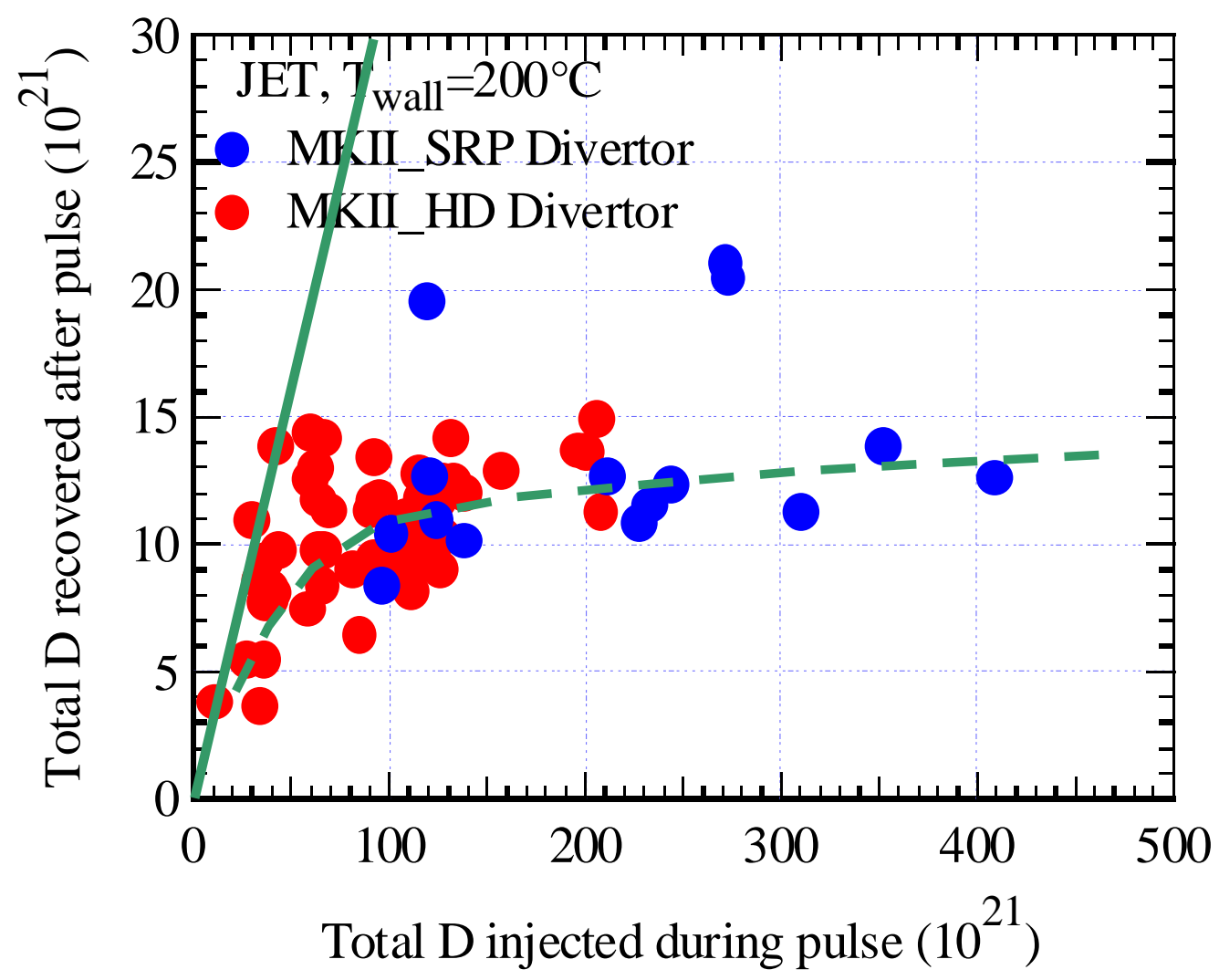

Figure 5: Total D recovered $600 \mathrm{sec}$ after the end of the pulse as a function of the total D injected during the pulse for a series of JET pulses with MKII_SRP and MKII_HD divertors 


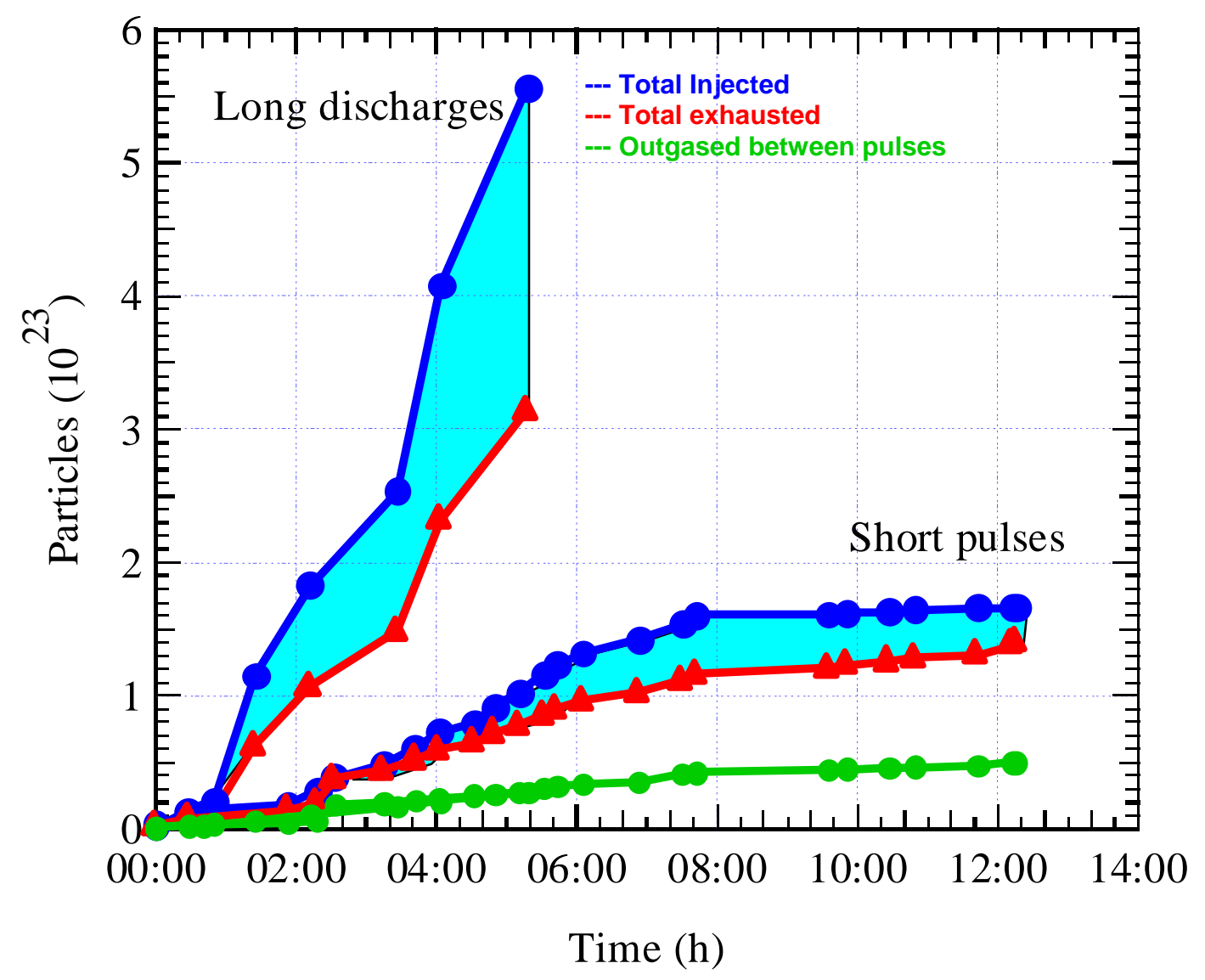

Figure 6: Integrated gas balance for a series of short pulses and for a series of long discharges. The out-gassed flux is plotted for the two series of experiments and they exhibit the same trend. 


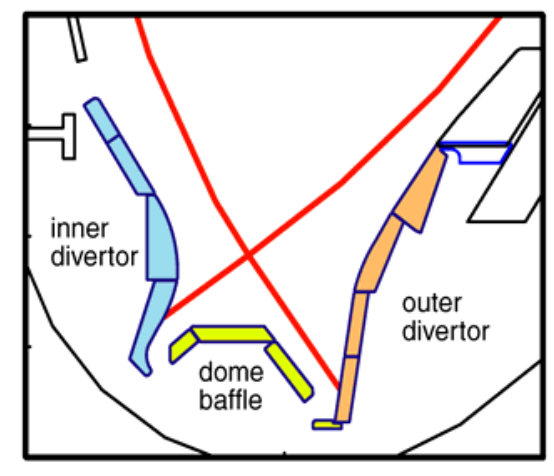

M Mayer et al.,

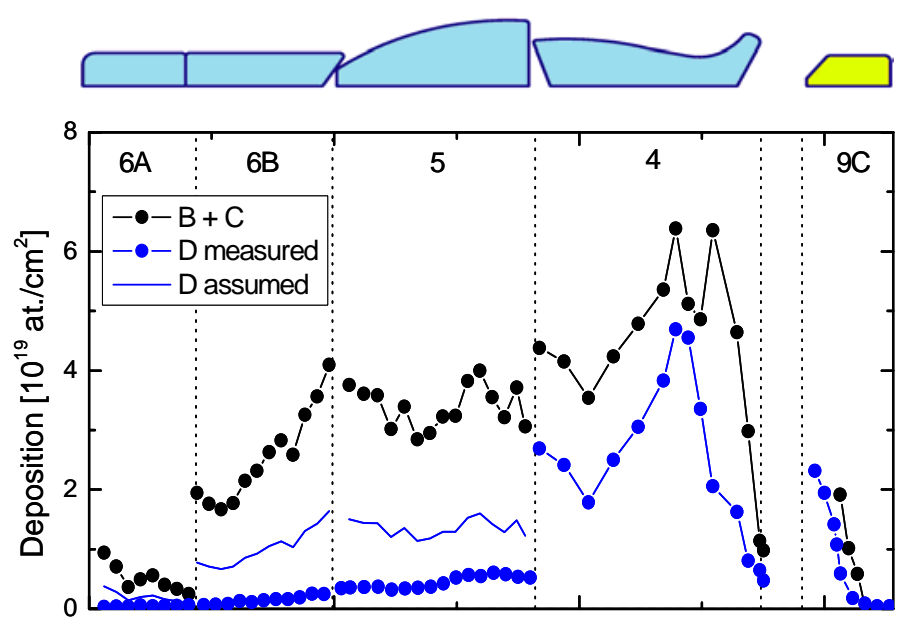

Figure 7: Deposition of D and C on the inner divertor of AUG for the 2002/2003 campaign [30]. 